\title{
Neural Involvement in Leprosy Treatment with Intra-Neural Injections of Prednisolone
}

\author{
T. H. TIO (TIONG HOO), m.d. (Amsterdam) \\ National Institute of Health, Surabaya (Indonesia)
}

Decompression of the thickened nerves has been known to relieve symptoms distal to the thickening. Surgical splitting of the neural sheath and transposing of the nerve as well as injections of corticosteroids have been used mainly for relieving pain. The writer has made some trials with intra-neural injections of Prednisolone (Benzon) as an auxiliary therapy in patients receiving oral anti-leprosy drugs. Most of these cases of neural involvement had shown a poor response to the sulphones. The writer wishes to present the results which he has obtained as they may be of interest to leprologists.

The trials which concerned a study of trophic changes of the skin, its appendages and muscles, could be carried out only for a short period, because the stock of Prednisolone was limited and could not be replenished.

\section{Identification of the involved nerve(s):}

This is fairly easy where the nerve is large, thickened and not embedded in a lot of subcutaneous fat. Pressure on the thickened nerve usually causes a sensation in the terminal area of distribution, thus helping in locating the nerve.

Selection of the correct site of injection is essential for obtaining maximal results and the site is determined according to the area of nervous disturbance; for instance if only the little finger is involved the injection should be given in the ulnar nerve; if all the fingers are involved injection should be in the brachial nerve. If the whole superior extremity including the scapular region is involved the site of injection would be the brachial plexus. The site of (presumably) maximal block in the case of easily palpable nerves can be determined by pressing along the nerve from the proximal towards the distal end and noting the severity of the sensation felt in the area of distribution. Pressure distal to the block causes markedly severe sensation as compared to the point proximal to the block.

When the thickening of the nerve is extensive (whether consecutive or of a beaded type) the most proximal thickening which still produces sensation on pressure is preferred by the writer. Without this sensation to guide the correct positioning of the needle one cannot be sure being in the sheath (see further under technique).

After injecting into the selected thickened segment effort is made to squeeze the drug along the course of the nerve. This can be assisted by positioning the limb to take advantage of gravity.

\section{Technique of injections:}

The thick nerve can easily be fixed either transversely or longitudinally between the thumb and forefinger. When the nerve is only slightly thickened or/and the subcutaneous fatty layer is substantial, the writer has the following technique:

After identifying the nerve it is pushed with the index and middle finger till fully stretched. When thus anchored the hypodermic needle is passed along the tip of the index finger into the nerve longitudinally. Confirmation of the correct insertion of the needle in the sheath is provided by a tingling sensation in the distal nerve ending on insertion or wiggling of the needle. This wiggling also moves the nerve and this can be felt by the finger. A much stronger and painful sensation is produced when the Prednisolone is actually injected.

Sometimes, however, there is complete absence of these sensory guides and in such cases the whole procedure is based on intelligent 
guesswork. Even in such cases after a few administrations clinical improvement can be observed (vide case $\mathrm{A}_{923} / 6_{5}$ and $\mathrm{Si}$ ) and even the tingling (sensory) phenomenon is restored. Gradually the tingling can be induced even by manipulating the nerve more and more proximally (vide case Ta).

Clinical improvements can be evaluated by comparison with the previous condition and/or the condition on the other side. For the skin and its appendages the points observed are:

- return of sensation (to cottonwool, pinprick, pulling out of hair).

- reduction of dryness and scaling; return of normal skin lustre, improvement of sweat and sebum production.

- arrest of loss of hair; resistance to epilation; regrowth of hair.

- normalization of nails.

For the muscles the points are:

- restoration of motor function.

- progressive increase in power.

- anatomical recovery of atrophy.

The patients were selected from two sources:

I. from the writer's private practice.

2. from the male out-patients of the National Institute of Health. The records exist of eight patients with functional involvement of the little finger (ulnar nerve) in whom intraneural Prednisolone injections were given for $\mathrm{I}$ to 3 weeks in a dosage of 5 to ro $\mathrm{mg}$. with good results in the sweat and return of sensation and in making the skin more lustrous, and improvement in the power of the interossei. The nerve also became thinner and softer:

I. B666/63 37y T-type of Leprosy of 5 years duration. 2 years unsuccessfully treated with sulphones. Very irregularly injected. Increase in pulling power little finger from $1900 \mathrm{Gr}$ * ${ }^{*}$ to $4 \mathrm{IOO}$ Gr. in 29 weeks.

2. Di $498 / 643^{8 y}$ Pure neural type of Leprosy of $3 \frac{1}{2}$ year duration.

In Institute for $\frac{1}{2}$ year unsuccessfully treated with sulphone (?previous therapy). After fairly regular injections increase of pulling power of little finger from 500 to I $700 \mathrm{Gr}$. in 22nd week.

3. Dirgo/64 28y T-type, later borderline type of leprosy of 3 years duration.
For 10 months in Institute treated with sulphones with no improvement (?previous treatment). Fairly regular injections increased pulling power of little finger from 1850 to $4900 \mathrm{Gr}$. in 2 ist week.

4. $\mathrm{N}_{322 / 65}$ 28y T-type of leprosy of 8 months duration. After I month sulphone therapy in Institute, fairly regular injections given. Increase pulling power of little finger from $700 \mathrm{Gr}$. to 1700 Gr. in 2 Ist week.

5. P974/63 igy L-type of leprosy of 8 years duration. 2 years' sulphone treatment in Institute gave no improvement. Pulling power of little finger improved from 1650 to $4500 \mathrm{Gr}$. in 22nd week after fairly regular injections.

6. Sir3o/62 40y T-type of leprosy of 6 years duration. 7 months' sulphone therapy in Institute (?previous therapy). Fairly regular injections increased pulling power little finger from 900 to $2300 \mathrm{~g}$. in $22 \mathrm{nd}$ week.

7. Si $\mathrm{S}_{40} 24 \mathrm{y}$ T-type of leprosy of $\mathrm{I}_{2} \frac{1}{2}$ years duration. No improvement at all after $i_{2}^{\frac{1}{2}}$ years' sulphone therapy. After fairly regular injections pulling power of little finger increased from 1000 to $3300 \mathrm{~g}$. in $27^{\text {th }}$ week.

One month after discontinuation of injection pulling power further increased to $3500 \mathrm{Gr}$. equal to pulling power of the other normal (?), little finger.

8. $\mathrm{S}_{756 / 65} 24 \mathrm{y}$ T-type of leprosy in reaction. Duration I year. One month after sulphone therapy in Institute irregularly injected. Increase in pulling power of little finger from 3050 to 4000 $\mathrm{g}$. in 20 th week.

SPECIAL PATIENTS

A. 923/65. male, 32 years, first examined $19 / 5 / 65$. Complaints: difficulty in movement of left leg; foot-drop, left, 4 months duration.

Previously unsuccessfully treated with B complex and thiamine by a neurologist.

Clinical findings left leg:

- atrophy of calf.

- skin relatively dry, scaly; touch and pain sensations lost; epilation of the sparse hair easy and painless.

- peroneal nerve thickened and insensitive.

Diagnosis: pure neural form of leprosy.

Therapy: patient placed on routine sulphone treatment.

$8 / 6$ no improvement noted.

$7.5 \mathrm{mg}$. Prednisolone (Benzon) injected into the peroneal nerve.

I5/6 anaesthesia now changed to paraesthesia specially on dorsum foot; movement of toes im-

*Throughout the paper Gr stands for grammes. 
proved. $7.5 \mathrm{mg}$. Prednisolone injected into peroneal nerve; the injection this time caused a sensation in the calf muscles.

22/6 pressure on peroneal nerve now c used sensation in calf muscles; epilation painful. $7.5 \mathrm{mg}$. Prednisolone I.N. given.

6/7 epilation difficult and painful; touch (cotton wool) and pinprick sensation returned. Sweating seen on the skin. Pressure on peroneal nerve and I.N. injection of $7.5 \mathrm{mg}$. Prednisolone now caused tingling in toes also.

27/7 further improvement noted. Skin now lustrous. Still unable to lift foot. $7.5 \mathrm{mg}$. Prednisolone I.N.

5/ Io improvement maintained, footdrop persistent.

Conclusion:

Rapid improvement of the trophic changes seen after Prednisolone injections I.N.

D. female, 3 I years, first examined July i 963 . Complaints :

1958: noticed fatigue right forearm and tremor of fingers after work. Noticed a discoloured spot on front right thigh. Unable to write in normal position of hand and forearm; could only write standing with forearm awkwardly pronated.

I96r: swinging of right arm while walking painful.

Unsuccessful electrotherapy by neurologist.

1963: Leprosy type $\mathrm{T}$ diagnosed.

Sulphones not tolerated (drug fever, giant urticaria). Put on Ciba 19o6. June r964 Additional Vadrine therapy started.

Jan. 1965: condition unchanged.

I 2.5 mg. Prednisolone I.N. into ulnar nerve started at weekly intervals. Decrease of tremor and ability to write in normal position gradually improved.

Feb.-Mar. I50 U Hyaluronidase (Benzon) added to $7.5 \mathrm{mg}$. Prednisolone I.N. injection. Faster improvement leading to return of normal functions. Ulnar nerve also returned to normal size. End of March I.N. injection stopped.

7/5 complaint of pain in right arm and scapular region. Put on weekly I.N. injections of $10 \mathrm{mg}$. Prednisolone +25 U Hyaluronidase subclavicularly into the brachial plexus.

28/5 Scapular pain disappeared. I.N. injections now given in the brachial nerve, $5 \mathrm{~cm}$. below the axilla; repeated weekly until $25 \mathrm{June}$, when patient left town.

5/6 Complaints of 'cramps' in right leg when walking. Femoral nerve found thickened and tender in the femoral triangle $5 \mathrm{mg}$. Prednisolone +25 U Hyaluronidase I.N. in femoral nerve; after three weeks 'cramps' disappeared, nerve restored to normal.
I 7/7 Patient re-examined: both right arm and right leg. N.A.D. Io $\mathrm{mg}$. Prednisolone $+25 \mathrm{U}$ Hyaluronidase I.N. into femoral and ulnar nerve. 2/8 Status quo ante; same injections given.

$5 / 9$ During past 5 weeks condition deteriorated. Complaints of 'cramps' in legs after walking long distance and difficulty in rising from sitting/ squatting position. Heavy feeling in right forearm; difficulty in writing. Ulnar nerve found to be markedly thickened. Femoral and peroneal nerve moderately thickened. The sciatic nerve could not be palpated satisfactorily. A.P.R. examination was done in order to feel the roots of the sciatic nerve. A considerably thickened nerve was felt at 6-9 o'clock position and pressure on this caused a painful tingling in the toes. Left side N.A.D. As the patient was not expected to come back for quite some time the following injections were given:

ulnar nerve: io $\mathrm{mg}$. Prednisolone $+50 \mathrm{U}$ Hyaluronidase. Peroneal and femoral nerve each: $7.5 \mathrm{mg}$. Prednisolone $+25 \mathrm{U}$ Hyaluronidase.

4/Io Patient had no complaints, all functions normal, all affected nerves felt thinner and softer. Ulnar and femoral nerves injected as on 5/9. R. sciatic nerve injected just below gluteal fold with Io mg. Prednisolone +25 U Hyaluronidase.

I4/ I I Marked improvement on all complaints. All thickened nerves and roots of sciatic nerves thinner and sof ter.

Remark: The exacerbation noted on 5/9 could perhaps be regarded as a 'rebound phenomenon'.

Ta. male, 38 years, first examined i $9 / 6 / 65$.

History: Jan. I96o after driving an LHD jeep in rain and cold, developed facial palsy left side with ectropion and drooping corner of mouth. Later an infiltrated red plaque developed around left eye. Diagnosed leprosy T-type with neural involvement. May i 96 I placed on Sulphone-chaulmogra (Lab. Roussel) injections.

In I959 was treated for TB lungs with streptomycin and I.N.H. First examined by writer $19 / 6 / 65$.

Clinical findings:

- no plaque seen

- ectropion, inability to close eyelids leaving lower $1 / 3$ cornea exposed, lacrimal overflow and some atrophy of orbital and cheek muscles.

- drooping corner mouth.

- thickened infra-orbital branch felt, no sensation on pressure.

- anaesthetic area lateral corner left eye.

Therapy: Sulphone-chaulmogra continued and Io $\mathrm{mg}$. Prednisolone I.N. given in the thickened nerve by feel alone. After three days marked improvement (22/6). 


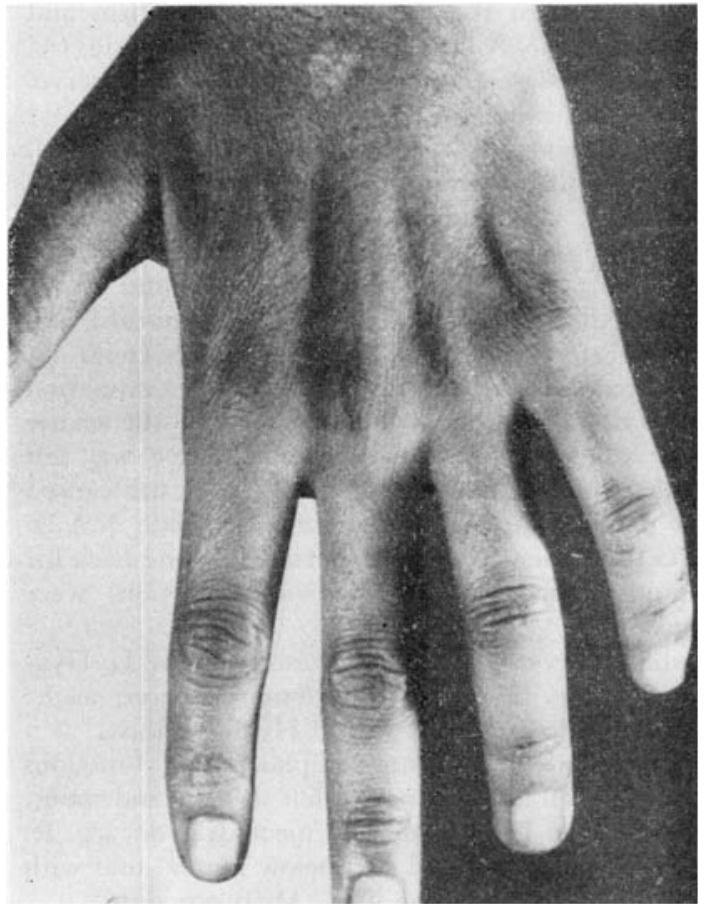

TABLE CASE $7, \mathrm{~S}_{14} 48 / 644 / \mathrm{V} / 65$

Note atrophy of interossei muscles (pulling power of little finger: $1000 \mathrm{Gr}$.)

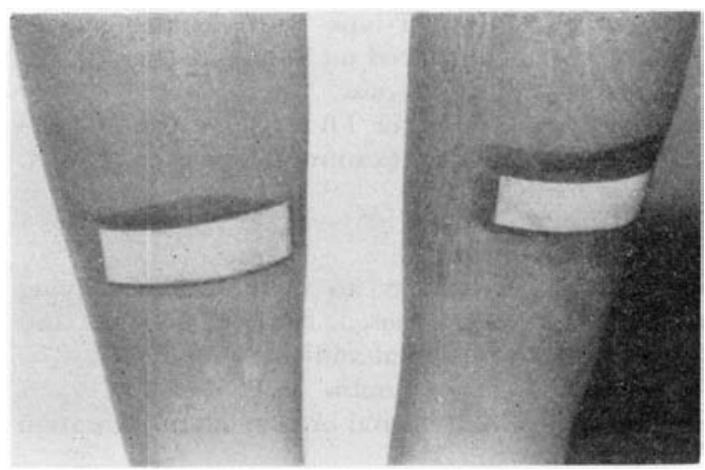

Case Si., 5/X/65

Skin of injected leg more shiny and less scaly; yet sweattest still negative as other leg.

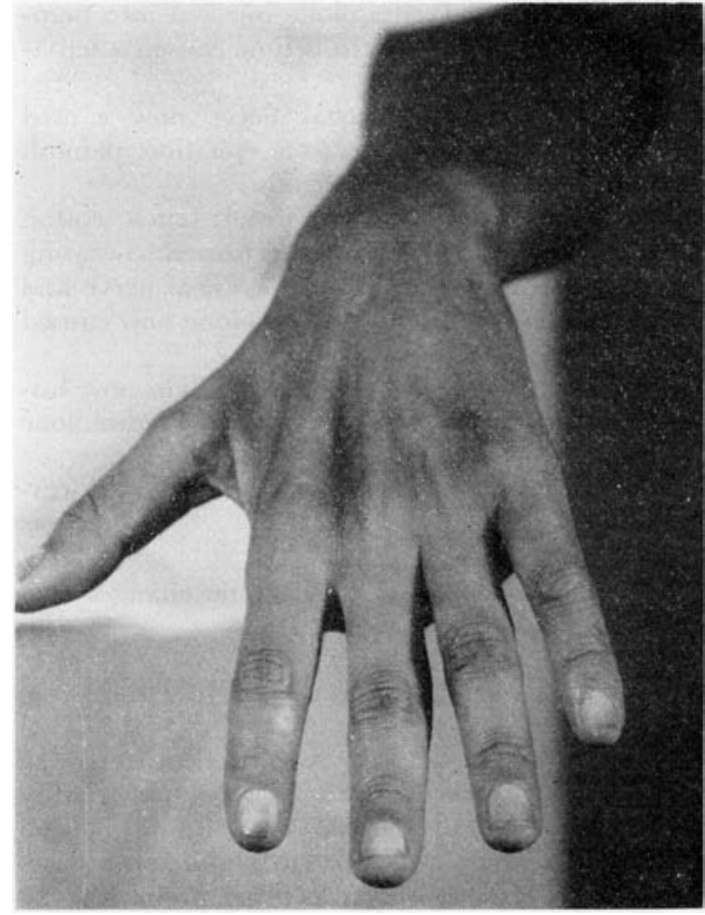

TABLE CASE $7, \mathrm{~S}_{14} 48 / 64$ 5/X/65

Note improvement of ulnar interossei muscles (pulling power of little finger: $3000 \mathrm{Gr}$.)

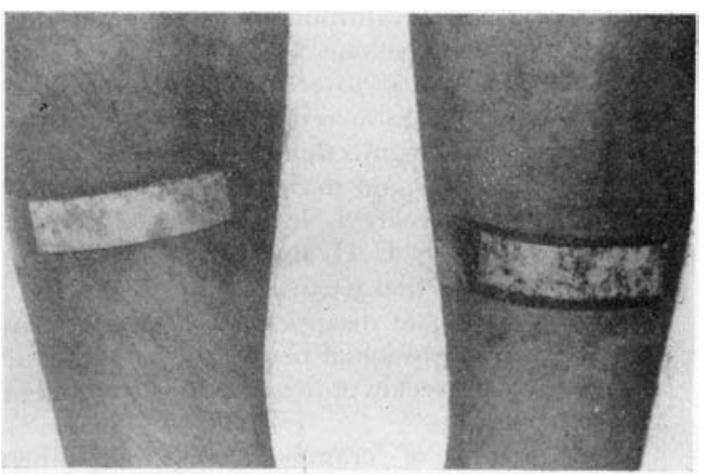

Case A 923/65 5/X/65

Rapid improvement of the skin of injected leg: now lustrous sweat-test positive 
- ability to close eyelid, now only the lower rim of cornea exposed; ectropion reduced; spill-over of tears reduced.

- sensation anaesthetic area returned.

- $7.5 \mathrm{mg}$. Prednisolone I.N.

$29 / 6$ Further reduction in lagophthalmos and other trophic disturbances; drooping of corner mouth reduced. Injection of $7.5 \mathrm{mg}$. Prednisolone into nerve produced tingling corners eye and mouth.

$6 / 7$ Further improvement of above; skin left face more lustrous, 7.5. mg. Prednisolone I.N. $7.5 \mathrm{mg}$. Prednisolone injection continued at weekly intervals. The return of terminal tingling on insertion of needle into nerve enabled the writer to give the injections into more and more proximal sites until on $20 / 7$ the injection site was $2 \mathrm{~cm}$. in front of ear.

24/8 The injection could be given into the facial nerve between the styloid process and the mandibular angle. Patient himself reported that he no longer needed to protect the eye against a breeze. Can now whistle.

7 \& $14 / 9$ injections as 24/8. Progressive improvement.

2I/9 Same dosage, given I.N. in foramen ovale, produced big shock on the left face.

Patient failed to turn up after $21 / 9$.

Si. female, 17 years, first examined $31 / 5 / 65$.

Complaints: foot-drop right side plus red, swollen toes.

Clinical findings:

Infiltrated maculae on face \& trunk + trophic changes on skin extremities.

Patient had been on Sulphones for $2 \frac{1}{2}$ years without results. This therapy was continued and $7.5 \mathrm{mg}$. Prednisolone was injected weekly into the vicinity of the peroneal nerve behind the head of the fibula (the nerve itself could not be located with any certainty) I.N. therapy was started on $3 / 6$.

Patient reported improvement soon after injection and her walk continued to improve.

After 2 weeks ( $17 / 6)$ clinical improvement as follows:

— skin less scaly

— toes less red and less swollen

- dragging of the right foot when walking reduced 28/8 skin condition further improved, pull on hair painful.

8/9 Peroneal nerve still could not be identified. Injections contined on $21 / 9,5 / 10$ and $12 / 10$ : foot drop persisting, Peroneal nerve still could not be identified.
PRELIMINARY GONCLUSIONS

I. Trophic disturbance of the skin and its appendages are favourably influenced even in patients which have failed to respond to the sulphones.

2. The duration of the acute phase (red bluish painful swelling of the fingers/toes) can be shortened.

3. As a result of the shortening of the acute phase loss of digits may be prevented.

4. Muscular power of atrophic muscles can be improved.

5. Lagophthalmos eventually leading to keratitis and blindness can be successfully treated.

6. Sudden and complete withdrawal seems to carry a risk of a rebound.

7. Additional Hyaluronidase appears to be of some benefit.

8. Sebaceous glands seem to restore their function earlier than sweat glands (see picture) as in the Hyfrecator-sparking treatment $\mathrm{x})$.

\section{S UMMAR Y}

Twelve patients with neural involvement were given intra-neural injections of Prednisolone and the results were favourable. It is suggested that this form of therapy is worth being tried out on a large scale.

REFERENCE

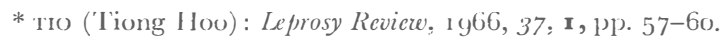

\section{A CKNOWLEGEMENTS}

I wish to thank Dr R. Wasito, Director of National Institute of Health, Surabaya, for his permission to use the facilities of the Institute. I am also grateful for the kind cooperation of Professor R. Goenawan and Dr R. Sardi. I feel greatly indebted to Dr V. S. Paranjpe for his valuable comments and assistance. 